\title{
Dynamics-aware Continuous-time Economic Dispatch: A Solution for Optimal Frequency Regulation
}

\author{
Roohallah Khatami \& \\ Masood Parvania \\ The University of Utah \\ roohallah.khatami@utah.edu \\ masood.parvania@utah.edu
}

\author{
Yu Christine Chen \\ The University of British Columbia \\ chen@ece.ubc.ca
}

\author{
Swaroop Guggilam \& \\ Sairaj Dhople \\ University of Minnesota \\ guggi022@umn.edu \\ sdhople@umn.edu
}

\begin{abstract}
This paper outlines a continuous-time economic dispatch (CTED) problem that intrinsically embeds dynamic constraints arising from the electromechanical behavior of synchronous generators and enables near-to-real-time optimal scheduling of generation. In its original form, the CTED problem is infinite-dimensional, however, we present a linear-programming reformulation that offers computational burden comparable to traditional economic dispatch. The resulting optimal dispatch trajectories are continuously differentiable and induce only small-signal variations in automatic generation control signals. In addition to yielding better system frequency response, this improves economic efficiency since the dispatch cost is better aligned with the actual cost of operating the system. We demonstrate the economic advantages and dynamic-performance improvements of the proposed method with time-domain simulations for a detailed differential algebraic equation model of an illustrative power network.
\end{abstract}

\section{Introduction}

The primary goal of real-time power system operation is to economically dispatch generation to meet net system load while regulating frequency. Presently, this is accomplished by modulating generator setpoints via a systematically engineered combination of: i) online proportional-integral control based on frequency and tie-line-flow errors (automatic generation control (AGC)); and ii) offline optimization that minimizes cost of generation based on the load forecast (economic dispatch (ED)). In this paper, we propose a continuous-time economic dispatch (CTED) problem and a companion solution algorithm, which reconciles the temporal gap that separates look-ahead dispatch and

This work was supported in parts by the National Science Foundation through grant 1453921, the Advanced Research Projects Agency-Energy under the Network Optimized Distributed Energy Systems program, and the Office of Naval Research through grant N000141812395. real-time control in an attempt to reduce generation cost, improve market efficiency, and enhance power quality.

Under prevailing practices, real-time markets do not systematically acknowledge frequency regulation provided by AGC as a market-based service [1]. By and large, a congestion-corrected version of incremental cost at the anticipated optimal operating point also dictates the cost of operating the system in real time $[2,3]$. This is tolerable and justifiable in networks dominated by high-inertia synchronous generators serving slow-varying loads. However, the retirement of synchronous generators and integration of grid-following power-electronics-interfaced renewable energy sources is resulting in faster, larger, and more frequent deviations away from synchronous operation $[4,5]$. Hence, there is a pressing need to accurately schedule generation over shorter time horizons so as to minimize the reliance on AGC regulation that-while engineered to encourage-does not guarantee economic optimality for large excursions away from ED setpoints. This aspect motivates the proposed continuous-time alternative to traditional ED. The formulated CTED problem incorporates pertinent dynamic constraints and yields dispatch trajectories that respect power balance at faster time scales, hence minimizing effort expended by AGC.

The existing architecture for frequency regulation comprising AGC and ED is illustrated in Fig. 1. While written accounts vary and practical implementations may differ, the consensus in technical literature is that classical ED is typically performed approximately every five minutes [6]. Optimizers of this problem present large-signal reference changes to AGC, which continually corrects synchronous-generator setpoints in an effort to match load in real time. The control effort exerted by AGC during the relatively long period of time that punctuates successive executions of ED may be significantly high given the expected outlook in contemporary power systems. Consequently, of the total accumulated cost over a given time period, the dispatch cost may be overshadowed by the cost of 


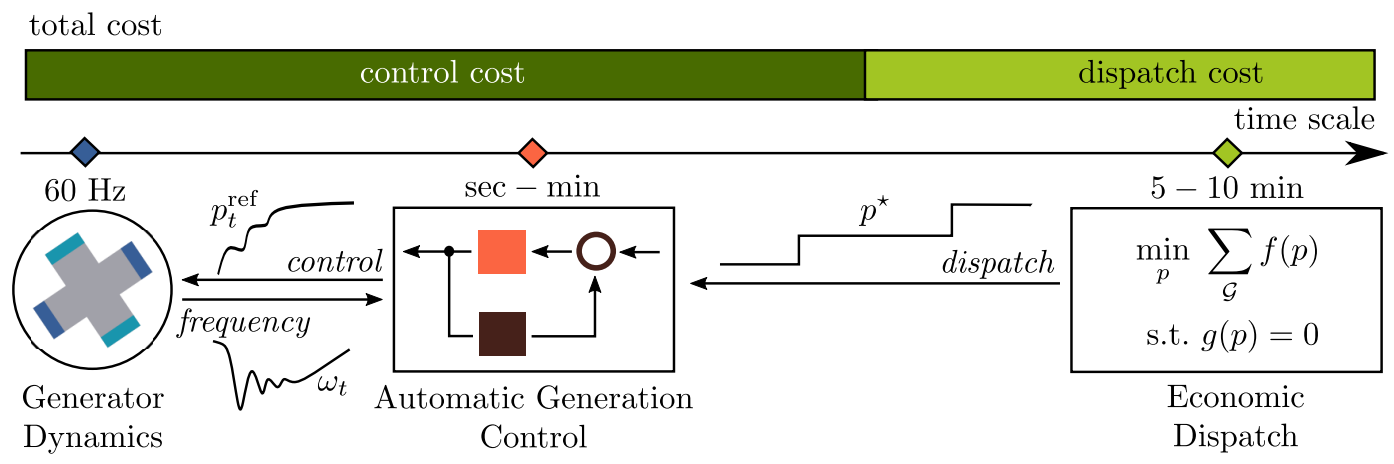

Figure 1. Prevailing architecture: ED is performed approximately once every five minutes. Dispatch signals are piece-wise constant and may involve large-signal changes. Cost of providing AGC-for which there is no established market-may constitute a significant portion of the total cost of operation.

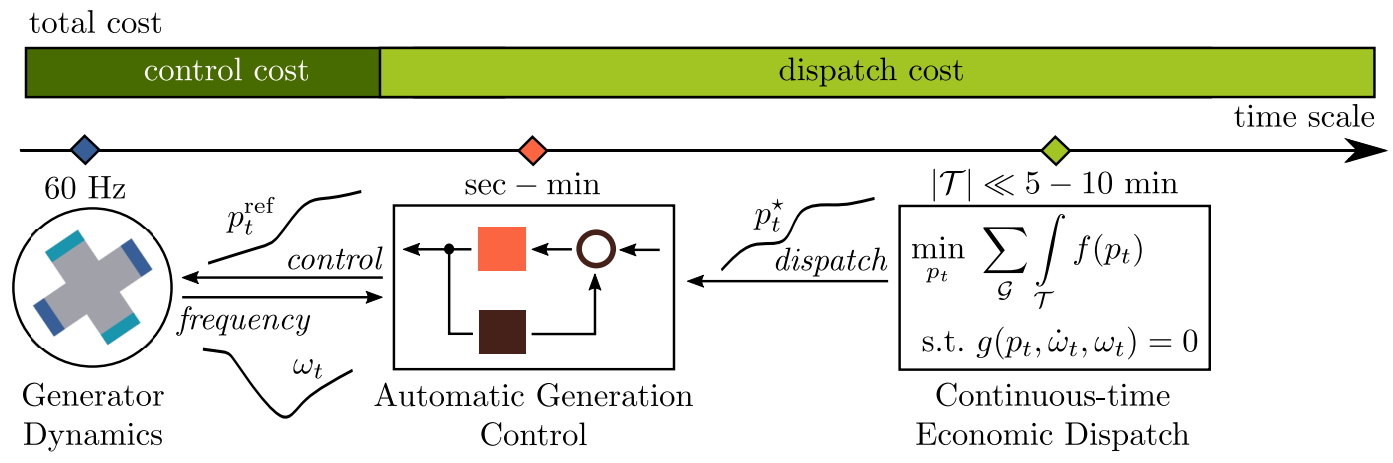

Figure 2. Proposed architecture: CTED is performed over a shorter scheduling horizon with fine-grained load samples. Dispatch signals are continuously differentiable. Cost of look-ahead dispatch-for which there is a well-established market-closely aligns with the total cost of operation. System frequency is better behaved since AGC control action does not involve aggressive changes in the generator setpoints.

effecting real-time frequency control. Now consider the proposed architecture sketched in Fig. 2. We put forth a continuous-time version of the conventional ED problem, with constraints that respect the impact of actuation on generator dynamics and system frequency; as a direct consequence, the scheduling horizon can be reduced. Optimal trajectories corresponding to this problem are continuously differentiable by design, rendering the corrective action to generator setpoints to be fine-grained. With this strategy, control effort exerted by AGC is lower, and the dispatch cost is more closely aligned with the actual operating cost of generators.

Realizing the architecture sketched in Fig. 2 requires effort in modeling (to tease out the dynamical constraints that are most pertinent) and computation (to solve the formulated infinite-dimensional variational CTED problem). We provide a brief overview covering these aspects next. To reflect underlying system dynamics in the CTED problem, we develop an aggregate model for system frequency in a given balancing area by suitably manipulating the swing dynamics of synchronous generators under a set of non-assumptive engineering-inspired approximations. From an algorithmic viewpoint, the proposed solution strategy to solve the formulated CTED problem is grounded in reducing the dimensionality of the continuous-time decision and parameter trajectories by representing them in a finite-order function space spanned by so-called Bernstein polynomials [7]. With this strategy, the resulting optimization problem boils down to a linear programming (LP) problem with computational complexity on par with conventional ED (typical realizations of which are LPs or linearly constrained quadratic programming problems).

Recognizing the latent potential to improve dynamic and economic performance, a variety of approaches have been put forth recently to temporally align economic dispatch with frequency regulation. A brief overview of pertinent recent literature is provided next. Primal-dual gradient methods are proposed in [8-12] to solve optimization problems that jointly consider generator scheduling and frequency regulation. 
A similar philosophy that encourages the unified optimization of AGC action and generator scheduling dictates the problems formulated in [13, 14], albeit, with differing solution strategies. Motivated by the goal of improving dynamic performance, some recent approaches have proposed embellishments to classical AGC $[15,16]$. We also bring to attention (a very wide body of) recent work on the design of feedback controllers that converge to the solution of cost minimization problems for power-system applications [17-24]. Finally, we point out the work in [25-27] where similar variational problems are solved with mixed-integer LP reformulations in the Bernstein polynomial function space for slower time-scale applications (unit commitment and incorporating ramping constraints in ED).

We conclude our introductory remarks by summarizing some features of the proposed method that also place its contributions in context of prior work highlighted above. First, we point out that the proposed approach to dispatch is aligned with current architectures (communication infrastructure and computation resources) and it does not fundamentally refashion either AGC or ED. Second, by minimizing AGC effort and aligning dispatch and total costs, we envision being able to efficiently allocate system capacity and dedicate reserves for operations at other time scales. Finally, we envision system frequency would be better behaved through large-signal variations in net load. This is because the solutions of the proposed CTED problem are restricted to be continuously differentiable trajectories that do not present large-signal changes to AGC references. Along these lines, we remark that the alternative of solving ED at faster time scales neither guarantees continuity of dispatch trajectories nor offers the option of capturing dynamic constraints across successive executions.

The remainder of this paper is organized as follows. In Section 2, we introduce the system dynamical model and obtain an aggregate frequency-response model for formulating the CTED problem. The CTED problem and its interoperability with AGC is discussed in Section 3. The function-space representation of all trajectories pertinent to the CTED problem is provided in Section 4. Finally, numerical simulations that demonstrate the improved economic efficiency and frequency response resulting from the proposed architecture are given in Section 5. Section 6 offers concluding remarks and directions for future work.

\section{System Dynamics}

This section introduces the synchronous-generator dynamical model and outlines the development of an aggregate representation of the generator dynamics that is then leveraged to design the CTED problem.

\subsection{Synchronous-generator Model}

We assume the power network is composed of $G$ generators indexed in the set $\mathcal{G}=\{1, \ldots, G\}$. For each generator $g \in \mathcal{G}$, let $\theta_{g}(t), \omega_{g}(t), P_{g}^{\text {mec }}(t)$, and $P_{g}^{\text {ele }}(t)$ denote the rotor angle, electrical angular frequency, turbine mechanical power, and electrical-power output, respectively. Assume each generator initially operates at the steady-state equilibrium point with $\omega_{g}(0)=$ $\omega_{\mathrm{s}}=2 \pi 60 \mathrm{rad} / \mathrm{s}$, the synchronous frequency. Defining $\Delta \omega_{g}:=\omega_{g}-\omega_{\mathrm{S}}$, pertinent dynamics of generator $g \in \mathcal{G}$ are described by the celebrated swing equations:

$$
\begin{aligned}
\dot{\theta}_{g}(t) & =\Delta \omega_{g}(t), \\
M_{g} \Delta \dot{\omega}_{g}(t) & =P_{g}^{\text {mec }}(t)-D_{g} \Delta \omega_{g}(t)-P_{g}^{\text {ele }}(t),
\end{aligned}
$$

where $M_{g}$ and $D_{g}$ denote, respectively, its inertia and damping constants. We emphasize that the above model is merely leveraged for analysis and development of the CTED problem. Validation through numerical simulations is performed with a detailed synchronous-generator model.

\subsection{Aggregate System Dynamical Model}

Assume that the effective impedances between nodes in the balancing area of interest are approximately the same, so that all generator speeds follow the same transient behaviour, i.e., $\Delta \omega_{g}=\Delta \omega$ in (1), $\forall g \in$ $\mathcal{G}[28,29]$. Then, the angular-frequency dynamics of each generator $g$ in (1) can be expressed as

$$
M_{g} \Delta \dot{\omega}(t)=P_{g}^{\mathrm{mec}}(t)-D_{g} \Delta \omega(t)-P_{g}^{\text {ele }}(t) .
$$

Summing (2) over all $g \in \mathcal{G}$ we get the following aggregate system dynamical model:

$$
\begin{aligned}
& M_{\mathrm{eff}} \Delta \dot{\omega}(t)=\sum_{g \in \mathcal{G}} P_{g}^{\mathrm{mec}}(t) \\
& -D_{\mathrm{eff}} \Delta \omega(t)-\sum_{g \in \mathcal{G}} P_{g}^{\mathrm{ele}}(t),
\end{aligned}
$$

where, we introduce the effective inertia constant, $M_{\mathrm{eff}}$ and the effective damping constant $D_{\text {eff }}$ below:

$$
M_{\mathrm{eff}}:=\sum_{g \in \mathcal{G}} M_{g}, \quad D_{\mathrm{eff}}:=\sum_{g \in \mathcal{G}} D_{g} .
$$


To facilitate subsequent discussion, we define:

$$
\begin{aligned}
P_{\mathcal{G}}^{\mathrm{mec}}(t) & :=\left[P_{1}^{\mathrm{mec}}(t), \ldots, P_{G}^{\mathrm{mec}}(t)\right]^{\mathrm{T}}, \\
P_{\text {load }}(t) & :=\sum_{g \in \mathcal{G}} P_{g}^{\mathrm{ele}}(t) .
\end{aligned}
$$

The nomenclature adopted to denote the sum of electrical-power outputs of the generators is indeed apt, since the summation is equal to the total system load along with losses. Leveraging these definitions, and denoting the $G$-dimensional column vector with all entries equal to unity by $1_{G}$, we can express (3) as:

$$
M_{\text {eff }} \Delta \dot{\omega}(t)=1_{G}^{\mathrm{T}} P_{\mathcal{G}}^{\mathrm{mec}}(t)-D_{\text {eff }} \Delta \omega(t)-P_{\text {load }}(t) .
$$

In effect, we remark that (7) is a point-wise-in-time power-balance constraint. For steady-state synchronous operation, we see that it indeed captures supply-demand balance, i.e., $1_{G}^{\mathrm{T}} P_{\mathcal{G}}^{\mathrm{mec}}=P_{\text {load }}$.

\section{Continuous-time Economic Dispatch}

In this section, we leverage the aggregate system dynamical model in (7) to derive the CTED problem formulation. We then compare and contrast the problem with classical ED. Finally, we provide a brief overview of how optimizers of (either) economic-dispatch routine are relayed to the synchronous generators via the AGC.

\subsection{Problem Formulation}

Denote the reference-power command issued by an economic-dispatch routine to generator $g$ at time $t$ by $P_{g}^{\text {ed }}(t)$, and collect instances $\forall g \in \mathcal{G}$ in the vector:

$$
P_{\mathcal{G}}^{\text {ed }}(t):=\left[P_{1}^{\text {ed }}(t), \ldots, P_{G}^{\text {ed }}(t)\right]^{\mathrm{T}} .
$$

The proposed CTED problem assumes the following form over scheduling horizon $\mathcal{T}$ :

$$
\begin{aligned}
\min _{P_{\mathcal{G}}^{\text {ed }}(t), t \in \mathcal{T}} & \int_{t \in \mathcal{T}} \sum_{g \in \mathcal{G}} C_{g}\left(P_{g}^{\text {ed }}(t)\right) d t \\
\text { s.t. } & 1_{G}^{\mathrm{T}} P_{\mathcal{G}}^{\text {ed }}(t)-D_{\text {eff }} \Delta \omega(t) \\
& -M_{\text {eff }} \Delta \dot{\omega}(t)=P_{\text {load }}(t), \\
& \underline{P}_{\mathcal{G}}^{\text {ed }} \leq P_{\mathcal{G}}^{\text {ed }}(t) \leq \bar{P}_{\mathcal{G}}^{\text {ed }}, \\
& \Delta \underline{\omega} \leq \Delta \omega(t) \leq \Delta \bar{\omega} .
\end{aligned}
$$

Above, $C_{g}(\cdot)$ is the cost function for generator $g \in \mathcal{G}$, and (9a) represents the accumulated cost of generation over the scheduling horizon. Notice that (9b) is a point-wise-in-time power-balance constraint that is inspired by and derives from (7), (9c) enforces box constraints on generator dispatch points over the scheduling horizon, and (9d) enforces box constraints on system frequency.

The optimal trajectories corresponding to the decision variables for the problem above, $P_{g}^{\text {ed } \star}(t), \forall g \in$ $\mathcal{G}, t \in \mathcal{T}$, act as inputs to AGC, which then yields reference values for the governors in the generators. We overview this in the forthcoming Section 3.3.

\subsection{Comparison with Classical Economic Dispatch}

The classical ED problem is of the following form:

$$
\begin{array}{ll}
\min _{P_{\mathcal{G}}^{\text {ed }}} & \sum_{g \in \mathcal{G}} C_{g}\left(P_{g}^{\text {ed }}\right) \\
\text { s.t. } & 1_{G}^{\mathrm{T}} P_{\mathcal{G}}^{\text {ed }}=P_{\text {load }}, \\
& \underline{P}_{\mathcal{G}}^{\text {ed }} \leq P_{\mathcal{G}}^{\text {ed }} \leq \bar{P}_{\mathcal{G}}^{\text {ed }} .
\end{array}
$$

In the problem above, (10b) enforces a power-balance constraint at each instant that the problem is solved. With slight abuse of notation, $P_{\text {load }}$ denotes the estimate of net system load at that instant. Box constraints are enforced as before, and the cost functions are the same as the ones in (9).

It is worth pointing out the synergies and points of departure of the problem in (9) and the classical dispatch problem expressed above in (10). We do so in a point-wise fashion and in no particular order next:

- The problem (9) is envisioned to be solved with fine-grained estimates of net system load over the scheduling horizon $\mathcal{T}$. On the other hand, classic ED in (10) is solved for pre-determined and isolated snapshots in time.

- Constraints (9b) and (10b) both capture power balance. While (9b) applies point-wise in time, it boils down to (10b) in steady state and for synchronous operation.

- Clearly, (9) is an infinite-dimensional problem. We propose a solution strategy that is grounded in projecting all continuous-time signals in an appropriate function space to facilitate computation. On the other hand, under mild assumptions on the cost function, (10) is a standard convex optimization problem.

- Even if problems (9) and (10) were solved with the same temporal granularity, optimizers of (9) would not be the same as those of (10). This is because $(10 \mathrm{~b})$ does not accurately capture power balance as does (9b). Also, there is no means of enforcing continuity on optimizers of (10). 
- The dual variable corresponding to the continuous-time power-balance constraint (9b) would reveal the real-time optimal marginal price of electricity. While analysis of dual problems corresponding to the conventional ED problem (10) is a mature field of study [6], a detailed analysis of the dual to the CTED problem (9) is beyond this paper's scope.

Given the discussion above, we hypothesize that the accumulated cost of scheduling generators would be much closer to the actual operating cost with the system dispatched by leveraging the optimizers of (9). The principal reason for this being that the control effort exerted by AGC would be minimized since power balance is enforced at faster time scales over the entire scheduling horizon. Furthermore, since the optimizers of (9) are engineered to be continuously differentiable trajectories (more on this in Section 4) and derived for a problem that acknowledges the underlying system dynamics (albeit only the swing equations), we also anticipate the system frequency response to be better behaved. We revisit both of these hypotheses in the simulation studies in Section 5.

\subsection{Integration with AGC}

Once the optimization problem (9) is solved, the optimal generator dispatch trajectories, $P_{g}^{\text {ed } \star}(t), \forall g \in$ $\mathcal{G}, t \in \mathcal{T}$ serve as inputs to AGC. For a single-area power system, the generator reference-power setpoints are obtained as [6]:

$$
P_{g}^{\mathrm{ref}}(t)=P_{g}^{\mathrm{ed} \star}(t)+\alpha_{g}\left(\xi(t)-\sum_{\ell \in \mathcal{G}} P_{\ell}^{\mathrm{ed} \star}(t)\right),
$$

where $\alpha_{g}$ denotes the Area Control Error (ACE) participation factor for generator $g \in \mathcal{G}$, and $\xi(t)$ is recovered from the following dynamics:

$$
\dot{\xi}(t)=\mathrm{ACE}-\xi(t)+\sum_{g \in \mathcal{G}} P_{g}^{\mathrm{ele}}(t) .
$$

The ACE for a single balancing area is given by

$$
\mathrm{ACE}=-\beta \frac{1}{|\mathcal{G}|} \sum_{g \in \mathcal{G}} \Delta \omega_{g}(t)
$$

where $\beta$ is the bias factor for the balancing area. While practical implementations may differ, classical references (see, e.g., [6]) suggest the following choices for bias factor and generator ACE participation factors:

$$
\beta=\sum_{g \in \mathcal{G}}\left(R_{g}^{-1}+D_{g}\right), \alpha_{g}=\frac{\left(I C_{g}^{\prime \star}\right)^{-1}}{\sum_{\ell \in \mathcal{G}}\left(I C_{\ell}^{\prime \star}\right)^{-1}} .
$$

Above, $R_{g}$ denotes the speed-droop regulation constant and $I C_{g}^{\prime \star}$ denotes the derivative of the optimal incremental cost of generation for generator $g$. While there is limited information in the literature on how this is to be interpreted and implemented during real-time operation, [6] advocates refreshing these every time an economic-dispatch problem is solved. For simplicity, in our simulations, we fix this to be the highest value among all possible incremental costs for the adopted piece-wise linear cost functions.

With classical ED, signals $P_{g}^{\text {ed } \star}$ in (11) would be constant between successive executions of the problem, and with the outlined continuous-time version of the problem, they would be continuously differentiable trajectories. We also remark that the AGC architecture discussed above is standard and remains unperturbed with our approach. We simply formulate a continuous-time counterpart (that acknowledges generator dynamics) to classical ED. The solution strategy to solve the infinite-dimensional problem (9) is discussed next.

\section{Function Space Representation of Continuous-time Economic Dispatch}

The proposed CTED problem in (9) is a constrained variational problem with an infinite-dimensional decision space, rendering it computationally intractable. Thus, instead of solving (9) directly, we leverage the approach outlined in $[25,27]$ to develop a scalable and efficient function space-based solution method for the proposed CTED problem. Central to the strategy is to model decision and parameter trajectories in a finite-order function space spanned by Bernstein polynomials. The Bernstein polynomials of degree $Q$ include $Q+1$ polynomials defined as:

$$
b_{q, Q}(t)=\left(\begin{array}{c}
Q \\
q
\end{array}\right) t^{q}(1-t)^{Q-q}, t \in[0,1]
$$

for $q \in \mathcal{Q}:=\{0, \ldots, Q\}$. Let us divide the scheduling horizon $\mathcal{T}$ into $N$ intervals of equal length $T$, and construct a set of basis functions in each interval $n$ using Bernstein polynomials of degree $Q$. Thus, the vector of basis functions spanning $\mathcal{T}$ :

$$
\mathbf{e}^{(Q)}(t)=\left[e_{1}^{(Q)}(t), \ldots, e_{P}^{(Q)}(t)\right]^{\mathrm{T}}
$$

contains $P=(Q+1) N$ components defined as:

$$
e_{n(Q+1)+q+1}^{(Q)}(t)=b_{q, Q}\left(\frac{t-t_{n}}{T}\right), t \in\left[t_{n}, t_{n+1}\right),
$$


for $n \in\{0, \ldots, N-1\}$ and $q \in \mathcal{Q}$. To simplify the notation, we define $p:=n(Q+1)+q+1$, and note that $p \in \mathcal{P}:=\{1, \ldots, P\}$.

With these fundamentals in place, we first discuss how the constraints and cost function in problem (9) can be expressed in the function space defined by Bernstein polynomials. Finally, in Section 4.4 we present the LP reformulation of the CTED problem (9).

\subsection{Modeling Power-balance (9b)}

Consider the system power balance constraint in (9b). Let us project $\Delta \omega(t), P_{\text {load }}(t)$, and entries of $P_{\mathcal{G}}^{\mathrm{ed}}(t):=\left[P_{1}^{\mathrm{ed}}(t), \ldots, P_{G}^{\mathrm{ed}}(t)\right]^{\mathrm{T}}$ in function spaces spanned by basis functions in (16) as follows:

$$
\begin{aligned}
& \Delta \omega(t)=\Delta \omega \mathbf{e}^{(Q)}(t), \quad \forall t \in \mathcal{T}, \\
& P_{\text {load }}(t)=\mathbf{P}_{\text {load }} \mathbf{e}^{(Q)}(t), \quad \forall t \in \mathcal{T}, \\
& P_{g}^{\text {ed }}(t)=\mathbf{P}_{g}^{\text {ed }} \mathbf{e}^{(Q)}(t), \quad \forall t \in \mathcal{T}, \forall g \in \mathcal{G} .
\end{aligned}
$$

Above, $\boldsymbol{\Delta} \omega, \mathbf{P}_{\text {load }}$, and $\mathbf{P}_{g}^{\text {ed }}$ are $P$-dimensional row vectors of Bernstein coefficients.

The time derivatives of Bernstein polynomials of degree $Q$ can be expressed as a linear combination of Bernstein polynomials of degree $Q-1$ [7], which allows us to define $\Delta \dot{\omega}(t)$ in the space spanned by Bernstein polynomials of degree $Q-1$ as:

$$
\begin{aligned}
\Delta \dot{\omega}(t) & =\Delta \omega \dot{\mathbf{e}}^{(Q)}(t)=\Delta \omega \mathcal{M} \mathbf{e}^{(Q-1)}(t) \\
& =: \Delta \dot{\omega} \mathbf{e}^{(Q-1)}(t), \quad \forall t \in \mathcal{T} .
\end{aligned}
$$

Above, $\mathcal{M}$ is the $P \times(P-N)$ matrix that relates $\dot{\mathbf{e}}^{(Q)}(t)$ and $\mathbf{e}^{(Q-1)}(t)$ [7], and $\Delta \dot{\omega}$ is the $(P-N)$-dimensional row vector of the Bernstein coefficients of $\Delta \dot{\omega}(t)$. From (21), the Bernstein coefficients of $\Delta \dot{\omega}(t)$ are linearly related to those of $\Delta \omega(t)$ as follows: $\Delta \dot{\omega}=$ $\Delta \omega \mathcal{M}$. Substituting the function space representation of $\Delta \omega(t), P_{\text {load }}(t), P_{g}^{\text {ed }}(t)$, and $\Delta \dot{\omega}(t)$ respectively from (18)-(20) and (21) and eliminating the Bernstein basis function vectors from both sides of the equality, the continuous-time power balance constraint in (9b) is converted to the following set of algebraic equations:

$$
M_{\text {eff }} \boldsymbol{\Delta} \omega \mathcal{M N}=\sum_{g \in \mathcal{G}} \mathbf{P}_{g}^{\text {ed }}-D_{\text {eff }} \boldsymbol{\Delta} \omega-\mathbf{P}_{\text {load }}
$$

where $\mathcal{N}$ is the $(P-N) \times P$ degree-raising matrix that relates $\mathbf{e}^{(Q-1)}(t)$ to $\mathbf{e}^{(Q)}(t)[7]$.

\subsection{Modeling Inequalities $(9 \mathrm{c})-(9 \mathrm{~d})$}

Due to the convex hull property of Bernstein polynomials, the projection coefficients form a control polygon that encompasses the continuous-time trajectories [7]. Thus, in order to project the continuous-time inequality constraints $(9 \mathrm{c})$ and $(9 \mathrm{~d})$ into the Bernstein function space, the Bernstein coefficients of the associated trajectories are confined to their minimum and maximum limits as below:

$$
\begin{aligned}
& \underline{P}_{g}^{\mathrm{ed}} 1_{P}^{\mathrm{T}} \leq \mathbf{P}_{g}^{\mathrm{ed}} \leq \bar{P}_{g}^{\mathrm{ed}} 1_{P}^{\mathrm{T}}, \quad \forall g \in \mathcal{G}, \\
& \Delta \underline{\omega} 1_{P}^{\mathrm{T}} \leq \Delta \omega \leq \Delta \bar{\omega} 1_{P}^{\mathrm{T}},
\end{aligned}
$$

where $1_{P}$ is a $P$-dimensional column vector of all ones.

\subsection{Modeling the Objective Function in (9a)}

Suppose a piece-wise linear cost function for generator $g$ with $S_{g}$ linearization segments. Let us associate positive auxiliary variable trajectories $\widehat{P}_{g, s}^{\text {ed }}(t)$ to each of the segments $s \in \mathcal{S}_{g}:=\left\{1, \ldots, S_{g}\right\}$. The dispatch trajectories of generators, as well as the associated cost functions, are cast in a linear form as below $\forall t \in \mathcal{T}, \forall g \in \mathcal{G}$ :

$$
\begin{aligned}
& P_{g}^{\mathrm{ed}}(t)=\underline{P}_{g}^{\mathrm{ed}}+\sum_{s \in \mathcal{S}_{g}} \widehat{P}_{g, s}^{\mathrm{ed}}(t), \\
& \widehat{C}_{g}\left(P_{g}^{\mathrm{ed}}(t)\right)=C_{g}\left(\underline{P}_{g}^{\mathrm{ed}}\right)+\sum_{s \in \mathcal{S}_{g}} \mu_{g, s} \widehat{P}_{g, s}^{\mathrm{ed}}(t),
\end{aligned}
$$

where $\widehat{C}_{g}\left(P_{g}^{\text {ed }}(t)\right)$ and $\mu_{g, s}$ respectively represent the linearized cost function of generator $g$ and the slope of its linearization segment $s$. In order to derive the function space representation of the linearized objective function, we first project auxiliary variables $\widehat{P}_{g, s}^{\text {ed }}(t)$ into the Bernstein function space as below $\forall t \in \mathcal{T}, \forall g \in \mathcal{G}$ :

$$
\widehat{P}_{g, s}^{\mathrm{ed}}(t)=\widehat{\mathbf{P}}_{g, s}^{\mathrm{ed}} \mathbf{e}^{(Q)}(t), \quad \forall s \in \mathcal{S}_{g},
$$

where $\widehat{\mathbf{P}}_{g, s}^{\text {ed }}$ represents the $P$-dimensional row vector of Bernstein coefficients confined to the lengths of linearization segments $\left|\widehat{P}_{g, s}^{\text {ed }}\right|$ as follows $\forall g \in \mathcal{G}$ :

$$
0 \leq \widehat{\mathbf{P}}_{g, s}^{\mathrm{ed}} \leq\left|\widehat{P}_{g, s}^{\mathrm{ed}}\right| 1_{P}^{\mathrm{T}}, \quad \forall s \in \mathcal{S}_{g}
$$

Substituting the function space representation of $P_{g}^{\text {ed }}(t)$ from (20) and of $\widehat{P}_{g, s}^{\mathrm{ed}}(t)$ from (27) in (25) and eliminating the vectors of basis functions from both sides, we can recast (25) as:

$$
\mathbf{P}_{g}^{\mathrm{ed}}=\underline{P}_{g}^{\mathrm{ed}} 1_{P}^{\mathrm{T}}+\sum_{s \in \mathcal{S}_{g}} \widehat{\mathbf{P}}_{g, s}^{\mathrm{ed}}, \quad \forall g \in \mathcal{G} .
$$


Finally, substituting (27) in (26), integrating it over the scheduling horizon $\mathcal{T}$, and summing over all generators, we can express (9a) as follows:

$$
\begin{aligned}
& \int_{t \in \mathcal{T}} \sum_{g \in \mathcal{G}} \widehat{C}_{g}\left(P_{g}^{\mathrm{ed}}(t)\right) d t \\
& =T \sum_{g \in \mathcal{G}} C_{g}\left(\underline{P}_{g}^{\mathrm{ed}}\right)+\sum_{g \in \mathcal{G}} \sum_{s \in \mathcal{S}_{g}} \mu_{g, s} \widehat{\mathbf{P}}_{g, s}^{\mathrm{ed}} \int_{t \in \mathcal{T}} \mathbf{e}^{(Q)}(t) d t \\
& =T \sum_{g \in \mathcal{G}}\left(C_{g}\left(\underline{P}_{g}^{\mathrm{ed}}\right)+\frac{1}{Q+1} \sum_{s \in \mathcal{S}_{g}} \mu_{g, s} \widehat{\mathbf{P}}_{g, s}^{\mathrm{ed}} 1_{P}\right)
\end{aligned}
$$

where the last line above follows from the identity [7]:

$$
\int_{t \in \mathcal{T}} \mathbf{e}^{(Q)}(t) d t=\frac{T}{Q+1} 1_{P} .
$$

\subsection{LP Reformulation of CTED in (9)}

In summary, the variational problem (9) is converted to an LP problem minimizing (30) subject to (22)-(24) and (28)-(29). The final version of the problem is:

$$
\begin{aligned}
& \min _{\mathbf{P}_{g}^{\text {ed }}, \widehat{\mathbf{P}}_{g, s}^{\text {ed }}, \Delta \omega} T \sum_{g \in \mathcal{G}}\left(C_{g}\left(\underline{P}_{g}^{\text {ed }}\right)+\sum_{s \in \mathcal{S}_{g}} \frac{\mu_{g, s} \widehat{\mathbf{P}}_{g, s}^{\text {ed }} 1_{P}}{Q+1}\right) \\
& \text { s.t. } \sum_{g \in \mathcal{G}} \mathbf{P}_{g}^{\mathrm{ed}}-\left(D_{\mathrm{eff}}+M_{\mathrm{eff}} \mathcal{M N}\right) \boldsymbol{\Delta} \omega=\mathbf{P}_{\text {load }} \\
& \mathbf{P}_{g}^{\mathrm{ed}}=\underline{P}_{g}^{\mathrm{ed}} 1_{P}^{\mathrm{T}}+\sum_{s \in \mathcal{S}_{g}} \widehat{\mathbf{P}}_{g, s}^{\mathrm{ed}}, \quad \forall g \in \mathcal{G}, \\
& 0 \leq \widehat{\mathbf{P}}_{g, s}^{\mathrm{ed}} \leq\left|\widehat{P}_{g, s}^{\mathrm{ed}}\right| 1_{P}^{\mathrm{T}}, \quad \forall g \in \mathcal{G}, s \in \mathcal{S}_{g}, \\
& \underline{P}_{g}^{\mathrm{ed}} 1_{P}^{\mathrm{T}} \leq \mathbf{P}_{g}^{\mathrm{ed}} \leq \bar{P}_{g}^{\mathrm{ed}} 1_{P}^{\mathrm{T}}, \quad g \in \mathcal{G}, \\
& \Delta \underline{\omega} 1_{P}^{\mathrm{T}} \leq \Delta \omega \leq \Delta \bar{\omega} 1_{P}^{\mathrm{T}}, \\
& \mathbf{P}_{g, k}^{\mathrm{ed}}=\mathbf{P}_{g, k+1}^{\mathrm{ed}}, k \in \mathcal{K}, \\
& \mathbf{P}_{g, k}^{\mathrm{ed}}-\mathbf{P}_{g, k-1}^{\mathrm{ed}}=\mathbf{P}_{g, k+2}^{\mathrm{ed}}-\mathbf{P}_{g, k+1}^{\mathrm{ed}}, k \in \mathcal{K}, \\
& \boldsymbol{\Delta} \omega_{k}=\boldsymbol{\Delta} \omega_{k+1}, k \in \mathcal{K}, \\
& \Delta \omega_{k}-\Delta \omega_{k-1}=\Delta \omega_{k+2}-\Delta \omega_{k+1}, k \in \mathcal{K} \text {. }
\end{aligned}
$$

Set $\mathcal{K} \subset \mathcal{P}$ introduced in constraints (32g)-(32j) captures points that interconnect different sub-intervals. These constraints ensure continuous differentiability of trajectories across adjacent sub-intervals.

\section{Simulation Results}

Case studies that compare the performance of the proposed CTED and classical ED are provided for the 9-bus transmission system shown in Fig. 3. Synchronous generators in this network are connected at buses $\mathcal{G}=\{1,2,3\}$, and loads are at buses $\{4,5,6,7,8,9\}$. Network- and generator-model parameters as well as generator cost functions are listed in the appendix. The network topology as well as the corresponding transmission-line and generator parameters are adopted from the Western System Coordinating Council (WSCC) network [30].

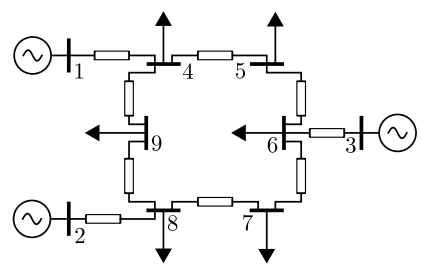

Figure 3. Single-area 3-generator 9-bus test system.

The optimization problems (10) and (32) are implemented in GAMS and solved using the CPLEX 12.6.2 solver [31]. Optimizers are then relayed to time-domain simulations performed with the Power System Toolbox (PST) [32]. Recall that the model introduced in Section 2.1 for the generator dynamics was primarily with the intention of formulating the CTED problem. In addition to the swing dynamics discussed in Section 2.1, our simulation includes a nonlinear differential algebraic equation model that considers lossy lines, turbine governor model, and a detailed two-axis machine model. A simulation period of 1 [min] is considered: the load takes values 230 [MW] and 430 [MW] during the first and last 20 [sec] intervals, and it grows linearly over $t \in$ $[20,40]$ [sec]. We compare the performance of four different scheduling strategies:

- $[1$ pt ED] Conventional ED (10) is performed only once at time $t=10$ [sec] over the 1 [min] simulation period.

- $[2 p t$ ED] Conventional ED (10) is performed twice at times $t=10,50$ [sec] over the $1[\mathrm{~min}]$ simulation period.

- $[3 p t$ ED] Conventional ED (10) is performed thrice at times $t=10,30,50[\mathrm{sec}]$ over the 1 [min] simulation period.

- $[\mathrm{CTED}]$ The CTED problem in (32) is solved with the simulation period divided into 5 sub-intervals. ${ }^{1}$

\footnotetext{
${ }^{1}$ The system load is approximated within each interval by Bernstein splines of degree $Q=5$. The projection coordinates of the system load's function space representation are derived by solving a least squares error problem [27].
} 
The extent of the load change in simulations is intended to be illustrative. The results demonstrate that with faster executions of conventional ED (i.e., going from $[1 \mathrm{pt} \mathrm{ED}]$ to $[3 \mathrm{pt} \mathrm{ED}])$, the dispatch cost is better aligned with the total cost. [CTED], on the other hand, ensures this alignment innately. We also demonstrate that conventional ED may result in poor dynamic performance if updates occur at instants when system dynamics are not in steady state (a likely scenario if the rate of executing ED is naïvely increased).

\subsection{Economic Performance}

Table 1 provides a breakdown of the total cost of operating the system into control and dispatch costs over the 1 [min] horizon with the four strategies highlighted

Table 1. Breakdown of accumulated total system operating cost into dispatch and control costs.

\begin{tabular}{|c|c|c|c|}
\hline Case & Control Cost [\$] & Dispatch Cost [\$] & Total Cost [\$] \\
\hline \hline$[1 \mathrm{ptED}]$ & 32.5703 & 66.7933 & 99.3636 \\
\hline$[2 \mathrm{ptED}]$ & 22.2502 & 77.0588 & 99.3091 \\
\hline$[3 \mathrm{ptED}]$ & 12.4967 & 87.1011 & 99.5978 \\
\hline$[\mathrm{CTED}]$ & 2.3040 & 96.9128 & 99.2170 \\
\hline
\end{tabular}

above. $[\mathrm{CTED}]$ provides the best alignment of dispatch cost with the total cost of operating the system. As the number of instances at which conventional ED is performed are increased, we also observe the trend that the control cost begins to constitute a smaller fraction of the total cost (implying that AGC is less stressed) and a majority of the total cost is the dispatch cost (implying improved accuracy of scheduling to meet load). The results above might suggest that as the number of executions of conventional ED is increased, we can expect economic performance on par with CTED. (That is, a better alignment of dispatch and total cost.) While this may indeed be the case, it can only be achieved with (potentially prohibitively) many executions of ED. In contrast, CTED is solved just once over the scheduling horizon. Furthermore, there is no guarantee on continuity and differentiability of optimizers of ED with increasing number of executions. We will examine the impact of this particular aspect on power quality and generator dynamics next.

\subsection{Dynamic Performance}

The dispatch schedules and resulting generator electrical output powers for [1ptED], [2ptED],

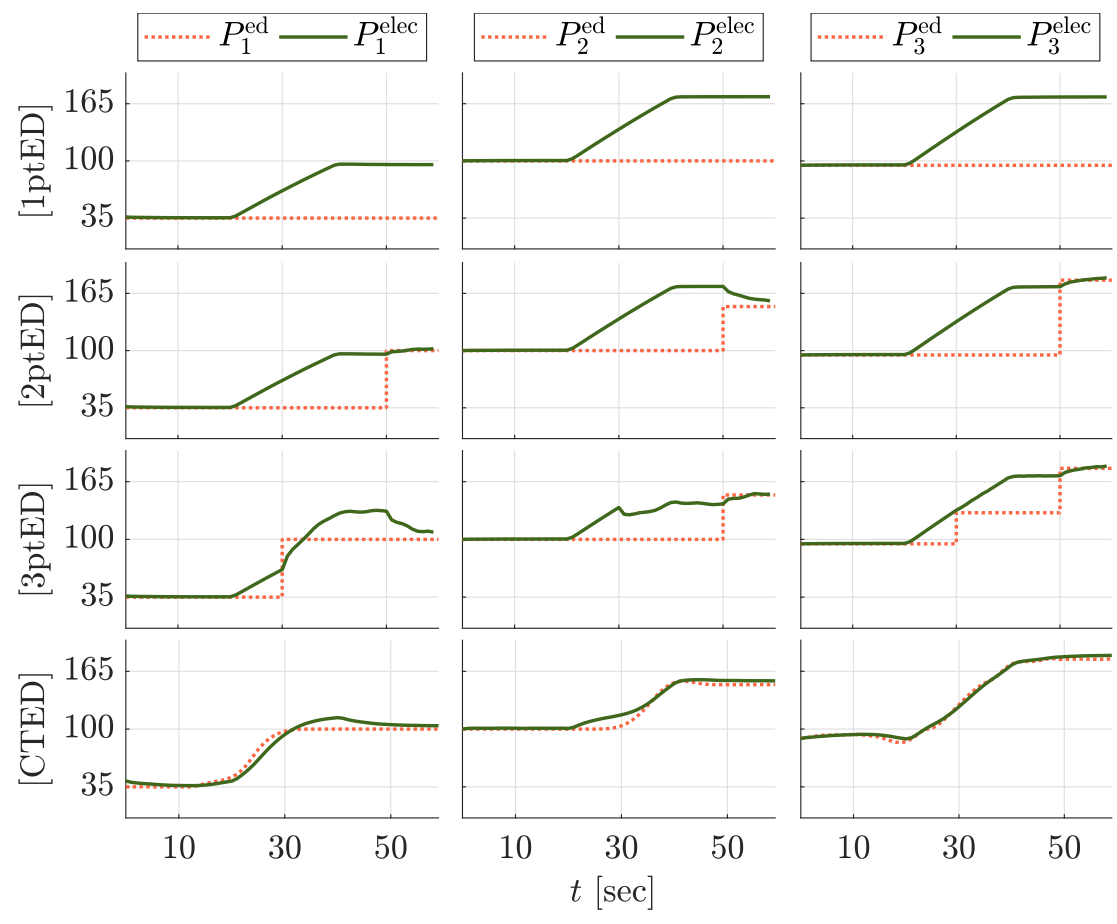

Figure 4. Generator electrical output-power $[\mathrm{MW}]$ values and solutions of optimization problems for the four dispatch strategies: $[1 \mathrm{ptED}],[2 \mathrm{ptED}],[3 \mathrm{ptED}]$, and $[\mathrm{CTED}]$. Notice that large-signal changes in dispatch induce undesirable transients in generator electrical output power with conventional ED for [1ptED], [2ptED], [3ptED]. In contrast, [CTED] yields improved dynamic performance. 


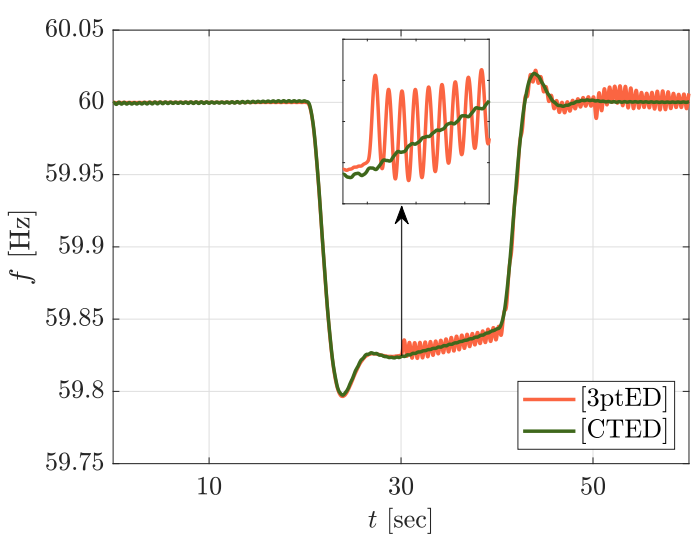

Figure 5. Electrical frequency recorded at the terminals of generator 1 obtained for the [CTED] dispatch strategy does not exhibit undesirable transients compared to [3ptED].

[3ptED], and [CTED] are plotted in Fig. 4. We can immediately appreciate the benefit of the dynamics-aware dispatch strategy. For all generators, we see a close match between the electrical output powers and the optimal dispatch schedules with [CTED]. The optimizers of conventional ED as obtained from [1ptED], [2ptED], and [3ptED] undergo large-signal changes with every execution: this ultimately translates to the electrical output powers of the generators exhibiting undesirable transients. In sharp contrast, the optimal dispatch trajectories as yielded by [CTED] are engineered to be continuously differentiable and anticipatory of system dynamics across the scheduling horizon. Consequently, the electrical output powers of the generators are also smoother and do not deviate significantly from the dispatch schedule. We plot the electrical frequency at the terminals of generator 1 through the simulation period in Fig. 5 for [CTED] and [3ptED] (trajectories corresponding to the other generators are similar, and therefore not shown). Undesirable transients in frequency with [3ptED] seen in Fig. 5 are due to large-signal changes in AGC setpoints initiated at a time when the system dynamics (particularly those attributable to generators) are not in steady state. On the other hand, the electrical frequency through the load transition with [CTED] is significantly better behaved.

\section{Concluding Remarks and Future Work}

This paper proposed a dynamics-aware continuous-time economic dispatch problem aligned with prevailing operational architecture in that it requires no major retrofits to existing communication infrastructure and computation resources. We demonstrated several benefits (from the perspectives of economics and power quality) over traditional economic dispatch. A function space-based solution method was leveraged to reduce the infinite-dimensional variational problem to a finite-dimensional LP problem to exercise available solvers. Time-domain simulations implemented in PST with accurate nonlinear differential algebraic equation models were provided to establish the virtues of the proposed method. Future work includes the addition of ramping constraints (on frequency and generator outputs), investigating the suitability of more representative higher-order dynamical models in the CTED formulation, consideration of frequency deviations caused by contingencies, and validating the idea in a network with multiple balancing areas.

\section{Appendix}

\subsection{Network and Dynamical-model Data}

Unless otherwise specified, all reported values are in per unit. The system base is $100[\mathrm{MVA}]$. The synchronous frequency, $\omega_{\mathrm{s}}=2 \pi 60\left[\mathrm{rad} \mathrm{sec}^{-1}\right]$. The generator damping coefficients are: $D_{1}=D_{2}=D_{3}=$ 1.28, inertia constants are: $M_{1}=M_{2}=M_{3}=$ 12.8 [sec], speed droop regulation constants are: $R_{1}^{-1}=$ $25, R_{2}^{-1}=25$ and $R_{3}^{-1}=25$ (i.e., $4 \%$ droop slope), turbine time constants are $\tau_{1}=\tau_{2}=\tau_{3}=5$ [sec]. Line parameters: $y_{14}=\mathrm{j} 0.0576, y_{45}=0.017+$ $\mathrm{j} 0.092, y_{56}=0.0 .39+\mathrm{j} 17, y_{36}=\mathrm{j} 0.0586, y_{67}=$ $0.0119+\mathrm{j} 0.1008, y_{78}=0.0085+\mathrm{j} 0.072, y_{28}=$ $\mathrm{j} 0.0625, y_{89}=0.032+\mathrm{j} 0.161$, and $y_{49}=0.01+\mathrm{j} 0.085$. Bias factor, $\beta=78.84$, ACE participation factors: $\alpha_{1}=$ $0.287, \alpha_{2}=0.345$ and $\alpha_{3}=0.368$.

\subsection{Generator Cost Functions}

All generators used in simulations have minimum and maximum limits of $35[\mathrm{MW}]$ and $200[\mathrm{MW}]$ respectively. Linearized cost functions with three linearization segments are adopted for all generators. Lengths and slopes of the segments are reported next.

Table 2. Linearized Cost-function Data.

\begin{tabular}{|c|c|c|c|c|c|c|}
\hline \multirow{2}{*}{ Generator } & \multicolumn{3}{|c|}{ Lengths [MW] } & \multicolumn{3}{c|}{ Slopes [\$/MWh] } \\
\cline { 2 - 7 } & $\left|\widehat{P}_{g, 1}^{\text {ed }}\right|$ & $\left|\widehat{P}_{g, 2}^{\text {ed }}\right|$ & $\left|\widehat{P}_{g, 3}^{\text {ed }}\right|$ & $\mu_{g, 1}$ & $\mu_{g, 2}$ & $\mu_{g, 3}$ \\
\hline \hline$g=1$ & 65 & 50 & 50 & 17.94 & 21.16 & 24.62 \\
\hline$g=2$ & 65 & 50 & 50 & 17.02 & 18.84 & 20.5 \\
\hline$g=3$ & 65 & 50 & 50 & 17.66 & 18.44 & 19.24 \\
\hline
\end{tabular}

\section{References}

[1] Z. Zhou, T. Levin, and G. Conzelmann, "Survey of US ancillary services markets," Argonne National Lab, Argonne, IL (United States), Tech. Rep., Jan 2016. 
[2] A. L. Ott, "Experience with PJM market operation, system design, and implementation," IEEE Transactions on Power Systems, vol. 18, no. 2, pp. 528-534, May 2003.

[3] R. Pedroncelli, "Frequency regulation compensation in the organized wholesale power markets-FERC 755," FERC Docket Nos RM11-7-000 and AD10-11-000, Tech. Rep., 2011.

[4] J. A. Taylor, S. V. Dhople, and D. S. Callaway, "Power systems without fuel," Renewable and Sustainable Energy Reviews, vol. 57, pp. 1322-1336, May 2016.

[5] F. Milano, F. Dörfler, G. Hug, D. J. Hill, and G. Verbic, "Foundations and challenges of low-inertia systems," in 2018 Power Systems Computation Conference, June 2018, pp. 1-25.

[6] A. J. Wood, B. F. Wollenberg, and G. B. Sheblé, Power generation, operation, and control. John Wiley \& Sons, 2013.

[7] P. Dierckx, Curve and surface fitting with splines. Oxford University Press, 1995.

[8] N. Li, C. Zhao, and L. Chen, "Connecting automatic generation control and economic dispatch from an optimization view," IEEE Transactions on Control of Network Systems, vol. 3, no. 3, pp. 254-264, September 2016.

[9] T. Stegink, A. Cherukuri, C. De Persis, A. v. d. Schaft, and J. Cortes, "Hybrid interconnection of iterative bidding and power network dynamics for frequency regulation and optimal dispatch," IEEE Transactions on Control of Network Systems, vol. 6, no. 2, pp. 572-585, June 2019.

[10] D. Cai, E. Mallada, and A. Wierman, "Distributed optimization decomposition for joint economic dispatch and frequency regulation," IEEE Transactions on Power Systems, vol. 32, no. 6, pp. 4370-4385, November 2017.

[11] Z. Wang, F. Liu, S. H. Low, C. Zhao, and S. Mei, "Distributed frequency control with operational constraints, part I: Per-node power balance," IEEE Transactions on Smart Grid, vol. 10, no. 1, pp. 40-52, January 2019.

[12] Z. Wang, F. Liu, S. H. Low, C. Zhao, and S. Mei, "Distributed frequency control with operational constraints, part II: Network power balance," IEEE Transactions on Smart Grid, vol. 10, no. 1, pp. 53-64, January 2019.

[13] M. Bazrafshan, N. Gatsis, A. F. Taha, and J. A. Taylor, "Coupling load-following control with OPF," IEEE Transactions on Smart Grid, vol. 10, no. 3, pp. 2495-2506, May 2019.

[14] G. Zhang, J. D. McCalley, and Q. Wang, "An AGC dynamics-constrained economic dispatch model," IEEE Transactions on Power Systems, 2019, to appear.

[15] D. Apostolopoulou, P. W. Sauer, and A. D. Domínguez-García, "Balancing authority area model and its application to the design of adaptive AGC systems," IEEE Transactions on Power Systems, vol. 31, no. 5, pp. 3756-3764, September 2016.

[16] Q. Liu and M. D. Ilić, "Enhanced automatic generation control (E-AGC) for future electric energy systems," in IEEE Power and Energy Society General Meeting, July 2012, pp. 1-8.

[17] M. Colombino, E. Dall' Anese, and A. Bernstein, “Online optimization as a feedback controller: Stability and tracking," IEEE Transactions on Control of Network Systems, 2019, to appear.
[18] Y. Tang, E. Dall'Anese, A. Bernstein, and S. H. Low, "A feedback-based regularized primal-dual gradient method for time-varying nonconvex optimization," in IEEE Conference on Decision and Control, December 2018, pp. 3244-3250.

[19] E. Dall'Anese, A. Bernstein, and A. Simonetto, "Feedback-based projected-gradient method for real-time optimization of aggregations of energy resources," in IEEE Global Conference on Signal and Information Processing, November 2017, pp. 1040-1044.

[20] A. Hauswirth, I. Subotić, S. Bolognani, G. Hug, and F. Dörfler, "Time-varying projected dynamical systems with applications to feedback optimization of power systems," in IEEE Conference on Decision and Control, December 2018, pp. 3258-3263.

[21] S. Menta, A. Hauswirth, S. Bolognani, G. Hug, and F. Dörfler, "Stability of dynamic feedback optimization with applications to power systems," in Annual Allerton Conference on Communication, Control, and Computing, October 2018, pp. 136-143.

[22] J. W. Simpson-Porco, B. K. Poolla, N. Monshizadeh, and F. Dörfler, "Quadratic performance of primal-dual methods with application to secondary frequency control of power systems," in IEEE Conference on Decision and Control, December 2016, pp. 1840-1845.

[23] A. Cherukuri and J. Cortes, "Distributed generator coordination for initialization and anytime optimization in economic dispatch," IEEE Transactions on Control of Network Systems, vol. 2, no. 3, pp. 226-237, September 2015.

[24] K. Hirata, J. P. Hespanha, and K. Uchida, "Real-time pricing and distributed decision makings leading to optimal power flow of power grids," in American Control Conference, July 2015, pp. 2284-2291.

[25] M. Parvania and A. Scaglione, "Unit commitment with continuous-time generation and ramping trajectory models," IEEE Transactions on Power Systems, vol. 31, no. 4, pp. 3169-3178, October 2015.

[26] M. Parvania and A. Scaglione, "Generation ramping valuation in day-ahead electricity markets," in $201649 \mathrm{th}$ Hawaii International Conference on System Sciences (HICSS), 2016, pp. 2335-2344.

[27] M. Parvania and R. Khatami, "Continuous-time marginal pricing of electricity," IEEE Transactions on Power Systems, vol. 32, no. 3, pp. 1960-1969, May 2017.

[28] M. D. Ilić and Q. Liu, Toward Sensing, Communications and Control Architectures for Frequency Regulation in Systems with Highly Variable Resources. New York, NY: Springer New York, 2012, pp. 3-33.

[29] S. S. Guggilam, C. Zhao, E. Dall'Anese, Y. C. Chen, and S. V. Dhople, "Optimizing DER participation in inertial and primary-frequency response," IEEE Transactions on Power Systems, vol. 33, no. 5, pp. 5194-5205, September 2018.

[30] A. R. Al-Roomi, "Power Flow Test Systems Repository," Halifax, Nova Scotia, Canada, 2015. [Online]. Available: https://al-roomi.org/power-flow

[31] The ILOG CPLEX, 2018. [Online]. Available: http: //www.ilog.com/products/cplex/

[32] J. H. Chow and K. W. Cheung, "A toolbox for power system dynamics and control engineering education and research," IEEE transactions on Power Systems, vol. 7, no. 4, pp. 1559-1564, November 1992. 